\title{
The effect of habitual and experimental antiperspirant and deodorant product use on the armpit microbiome
}

Julie Urban, Daniel J Fergus, Amy M Savage, Megan Ehlers, Holly L Menninger, Robert R Dunn, Julie E Horvath

An ever expanding body of research is investigating the human microbiome in general and the skin microbiome in particular. Microbiomes vary greatly from individual to individual. Understanding the factors that account for this variation, however, has proven challenging, with many studies able to account statistically for just a small proportion of the interindividual variation in the abundance, species richness or composition of bacteria. The human armpit has long been noted to host a high biomass bacterial community, and recent studies have highlighted substantial inter-individual variation in armpit bacteria, even relative to variation among individuals for other body habitats. One obvious potential explanation for this variation has to do with the use of personal hygiene products, particularly deodorants and antiperspirants. Here we experimentally manipulate product use to examine the abundance, species richness, and composition of bacterial communities that recolonize the armpits of people with different product use habits. In doing so, we find that when deodorant and antiperspirant use were stopped, culturable bacterial density increased and approached that found on individuals who regularly do not use any product. In addition, when antiperspirants were subsequently applied, bacterial density dramatically declined. These culture-based results are in line with sequence-based comparisons of the effects of long-term product use on bacterial species richness and composition. Sequence-based analyses suggested that individuals who habitually use antiperspirant tended to have a greater richness of bacterial OTUs in their armpits than those who use deodorant. In addition, individuals who used antiperspirants or deodorants long-term, but who stopped using product for two or more days as part of this study, had armpit communities dominated by Staphylococcaceae, whereas those of individuals in our study who habitually used no products were dominated by Corynebacterium. Collectively these results suggest a strong effect of product use on the bacterial composition of armpits. Although stopping the use of deodorant and antiperspirant similarly favors presence of Staphylococcaceae over Corynebacterium, their differential modes of action exert strikingly different effects on the richness of other bacteria living in armpit communities. 
2 Julie M. Urban ${ }^{1}$, Daniel J. Fergus ${ }^{1}$, Amy M. Savage ${ }^{2}$, Megan Ehlers ${ }^{1,2}$, Holly L. Menninger ${ }^{3}$,

3 Robert R. Dunn ${ }^{4}$, Julie E. Horvath ${ }^{1,5,6}$

4

5 1-North Carolina Museum of Natural Sciences, Raleigh, NC, USA 27601

6 2- Department of Biology \& Center for Computational \& Integrative Biology, Rutgers

7 University-Camden, Camden, NJ 08103

8 3- Department of Biological Sciences, North Carolina State University, Raleigh, NC, USA

927695

10 4-Department of Applied Ecology and Keck Center for Behavioral Biology, North Carolina State

11 University, Raleigh, NC, USA 27695

12 5-Department of Biological and Biomedical Sciences, North Carolina Central University,

13 Durham, NC, USA 27707

14 6- Department of Evolutionary Anthropology, Duke University, Durham, NC, USA 27708

15

16 Corresponding author:

17 Julie E. Horvath

18 North Carolina Museum of Natural Sciences

1911 West Jones St.

20 Raleigh, NC, USA 27601

$21 \quad 919-707-8242$

22 julie.horvath@naturalsciences.org 
25 Keywords: Microbiome, axilla, armpit, biodiversity, antiperspirant, deodorant 26 


\section{Introduction}

Like the gut or the mouth, the human skin is covered with life. This life includes bacteria,

29 fungi, Archaeans, bacteriophages, and even animals such as nematodes and Demodex mites

30 (Marples, 1965; Grice \& Segre, 2011; Kong \& Segre, 2012). Since the 1950s it has been clear

31 that the precise composition of the skin biome influences its effectiveness as a defensive layer

32 against pathogens (Eichenwald et al., 1965), and contributes to bodily odors (Shelley, Hurley \&

33 Nicholas, 1953). Some species are better at defending our skin than others (Christensen \&

34 Brüggemann, 2014), just as some species produce different odors than do others (Leyden et al.,

35 1981). What is unclear is the extent to which human behaviors influence the composition of skin

36 microbes. Inasmuch as two types of products, antiperspirants and deodorants, are used daily in

37 armpits by a large number of people (perhaps as many as $90 \%$ of people in the US, according to

38 Benohanian, 2001), armpits represent an interesting context in which to explore the general

39 phenomenon of how human behavior and product use influence skin microbes.

A long history of work focuses on the biology of the culturable bacteria in armpits

(Shelley, Hurley \& Nicholas, 1953; Marples, 1965; Leyden et al., 1981). More recent work has

built upon this history to consider both those taxa that are culturable and those whose presence is only detectable (to date) through sequencing. In one of the first of this new wave of studies,

44 Grice et al. (2009) sampled, cloned, and Sanger-sequenced bacteria from 20 body regions sampled from nine participants. The most prominent bacteria present in armpits were species of Corynebacterium, Staphylococcus, Betaproteobacteria, Clostridiales, Lactobacillus, Propionibacterium, and Streptococcus. Interestingly, bacterial residents of armpits were shown to be highly variable even across this small number of participants: four participants' communities were dominated by Corynebacterium species, three by Staphylococcus species, and 
50 two by Betaproteobacteria. Gao et al. (2010) also found large variation among individuals in the

51 composition of armpit bacterial taxa (drawn from similar genera as in Grice et al., 2009). This

52 high person-to-person variability stands in contrast to samples from other skin habitats, which

53 show less inter-individual variation, and are locations where product use is less common

54 (Costello et al., 2009; Caporaso et al., 2011; Hulcr et al., 2012) (although see Callewaert et al.,

55 2013). This variability might simply reflect stochastic effects or even the sequencing of dead

56 bacteria on the skin (Grice \& Segre, 2011). However, Egert et al. (2011) found that most of the

57 same common taxa in the armpits, including most/all of the common taxa found in Grice et al.

58 (2009) and Gao et al. (2010), were the most metabolically active and contributed the most to

59 armpit odor.

60 The high variability in armpit communities among individuals suggests that an

61 unaccounted for factor, perhaps product use, might be exerting a strong influence on armpit

62 bacteria, which may in turn have functional consequences for the host. Older, culture based

63 studies suggest that the use of deodorants and antiperspirants appears to reduce the abundance of

64 culturable bacterial taxa, particularly those of Corynebacterium, a slow-growing lineage of

65 bacteria that plays a key role in the production of armpit odor (Leyden et al., 1981). This effect is

66 not accidental inasmuch as the intent of underarm products has long been the reduction in armpit

67 odor either through direct reduction in the biomass of bacteria, or through blocking the exudates

68 of the apocrine glands, which become odiferous when metabolized by bacteria (Taylor et al.,

69 2003; Wilke et al., 2007, Fredrich et al., 2013). Second, two recent studies by Callewaert et al.

$70(2013 ; 2014)$ found an association between product use and the diversity of bacteria in armpits.

71 Although the Callewaert et al. (2014) study that specifically tested for effects of product use in

72 nine people did not include a control group (people who habitually do not use any products), this 
73 work is clearly suggestive of a potentially large impact of underarm products on entire

74 communities of armpit bacteria.

75 Here, we examine several questions related to product use and armpit bacterial

76 communities. First, we test whether there is a direct relationship between the abundance of

77 readily culturable bacteria and product use. Second, we use a sequencing based approach to

78 consider whether there are long-term differences in the species richness and composition of

79 armpit bacteria on individuals who habitually use antiperspirant, deodorant or no product. We

80 also consider whether these differences are in line with what would be expected given the

81 intended effects of underarm products in reducing the abundance of odor-causing armpit bacteria

82 (i.e., primarily Corynebacterium), as well as the different mechanisms by which antiperspirants

83 versus deodorants achieve this reduction. Finally, we compare the abundance of two focal taxa,

84 an OTU categorized as Staphylococcaceae (predominant in comparison to the OTU categorized

85 as Staphylococcus) and an OTU of Corynebacterium, as a function of product use, gender (based

86 on Callewaert et al.'s, 2013 work), and time since ceasing product use.

\section{Materials and Methods}

\section{Participants}

Eighteen individual citizen scientists (public participants in scientific research) were

91 recruited through the NC Museum of Natural Sciences Genomics \& Microbiology Lab for

92 armpit community sampling. Prior to the start of the experiment, the proposed study was

93 reviewed and approved by the North Carolina State University Institutional Review Board for

94 the Use of Human Subjects in Research (IRB\#1987). All participants were supplied with an IRB

95 authorized consent form and all provided their consent to participate (indicated by their 
96 signatures) prior to the start of the experiment. Individuals were recruited to represent three

97 groups, each with equal numbers of men and women. One group of participants reported

98 typically not using any deodorants or antiperspirants. Another group reported regular use of

99 deodorant-only products. The third group reported regular use of antiperspirant products. Not

100 everyone in our study used the same product brand, but all antiperspirant users used products

101 containing aluminum zirconium trichlorohydrex Gly as the active ingredient. Although we

102 designed our study to have equal group sizes, participant drop-out and product mis-classification

103 during the course of the study resulted in a final sample of five participants each in the no

104 product use (three men and two women) and deodorant-only use (three men and two women)

105 groups, and seven participants (three men and four women) who used antiperspirant-containing

106 products.

107 We experimentally altered product use by the participants over eight days. On the first

108 day (Day 1), participants went about their normal habits (e.g. applying deodorant or

109 antiperspirant if they normally wore it); we did not require that they apply product at any certain

110 time of day or number of times per day, nor did we require that they shower a certain amount.

111 All participants indicated showering/bathing 3-14 times per week (Supplemental Table 1), and

112 they were asked to continue their normal showering/bathing routine for this experimental week.

113 On Days 2-6, participants were asked to discontinue product usage. During the last two days

114 (Days 7 and 8) of sampling, all individuals, including those who did not normally use

115 antiperspirant or deodorant, were asked to use an antiperspirant/deodorant product (we provided

116 Secret Powder Fresh for women and Old Spice Fiji for men-both contained aluminum zirconium

117 trichlorohydrex Gly as the active ingredient). On each of the eight days of the study, both armpits

118 of each individual were swabbed once for 45-60 seconds between 11am (EST) and 1pm (EST) 
119 with a dual-tipped sterile BBL ${ }^{\mathrm{TM}}$ CultureSwab ${ }^{\mathrm{TM}}$ (Becton Dickinson and Company, Franklin

120 Lakes, NJ). A tradeoff exists between sampling participants many times (daily) over a short

121 period of time and sampling less frequently over a longer period of time (weeks to months). We

122 opted for more frequent sampling (daily for eight days) versus longer-term sampling to assess

123 shorter-term effects of ceasing product use in order to achieve a balance across competing

124 considerations including participant compliance, available budget, supplies and personnel time.

\section{Culture-based Sampling}

126 Bacteria sampled from the left and right armpits were cultured the same day they were

127 collected by immersing one of the two sample swabs into $0.5 \mathrm{~mL}$ of phosphate buffered saline

128 (PBS), mixing, and spreading $20 \mu \mathrm{l}$ of this solution onto sterile LB agar plates. All cultures were

129 incubated aerobically at $37^{\circ} \mathrm{C}$ for approximately 22 hours, and then stored at $4^{\circ} \mathrm{C}$ to stop further

130 colony growth. Plates were photographed and numbers of colonies occupying a standard central

131 region of each plate $\left(18.07 \mathrm{~cm}^{2}\right)$ was counted using Image $\mathrm{J}$ software (version 1.46 , Rasband,

132 1997-2014). The $18.07 \mathrm{~cm}^{2}$ region on the plate does not necessarily equate to the same size

133 region on a person's skin. Abundance counts for bacteria cultured from the left versus right

134 armpit were averaged to yield one abundance score (i.e., average number of CFU present on two

135 culture plates) per person for each day of the experiment.

\section{Culture-based Analyses}

To test the effects of time and change in product use on abundance of culturable bacteria,

138 we ran several analyses of variance (ANOVA) on time intervals of interest, using SPSS version

13921.0 (IBM Inc., USA). To determine whether regular use of underarm products exerts an effect

140 on initial abundance immediately following disuse of products, we ran a one-way ANOVA on

141 abundance of culturable bacteria sampled on Day 2 (the first day all subjects ceased use of 
142 products). We conducted mixed-model (day x product use x gender) ANOVAs to test effects of

143 stopping product use and continued disuse on abundance of culturable bacteria (Day 1 versus

144 Day 2-6), and to test effect of antiperspirant application on abundance (Day 6 versus Days 7-8).

\section{Sequence-based Sampling}

146 Several extensive surveys of armpit microbes (e.g., Flores et al.2012) and our personal

147 observations indicate that armpit samples show poor amplification for sequence-based analyses.

148 In addition, our preliminary experiments indicated that presence of product (antiperspirant or

149 deodorant) inhibited our ability to isolate and subsequently PCR amplify armpit bacterial DNA,

150 so we chose to conduct our "early" sequence-based sampling on Day 3 (the second day of

151 continued product disuse) and the "late" sampling on Day 6 (the fifth and final day of continued

152 product disuse). The expectation was that on Day 3, residual effects of product use would remain

153 persistent (if they existed) and that by Day 6 bacterial populations might have begun to recover

154 from whatever such effects might be, and hence converge on a common, shared composition.

155 DNA was isolated from the second sample swab, stored dry at $-20^{\circ} \mathrm{C}$ for up to one month

156 prior to isolation, using the PowerSoil DNA Isolation Kit (MO BIO Laboratories, Carlsbad, CA)

157 with minor modifications. Instead of using soil, the swab tip was swirled into the beads for

158 approximately 5 seconds and removed before adding solution C1. For the elution, solution C6

159 was heated to $50^{\circ} \mathrm{C}$ before being added in the final step of the protocol. Also, only $50 \mu 1$ of

160 solution C6 was used to elute the DNA. All isolated DNA samples were stored at $-20^{\circ} \mathrm{C}$. The V4

161 region of 16S rDNA was PCR amplified from each DNA sample using Premix ExTaq (Takara

162 Bio) with primers designed to amplify bacteria and Archaea (515F:

163 GTGCCAGCMGCCGCGGTAA, and 806R: GGACTACHVGGGTWTCTAAT), modified to

164 include Roche 454 adapters and index sequences as previously described (Hulcr et al., 2012). 
165 Reactions were set up such that there was a unique index for each subject, arm (left vs. right) and

166 day. All PCRs, including a no-template control, were performed in triplicate and after

167 thermocycling, each triplicate was pooled and purified using an UltraClean-htp 96-well PCR

168 Clean-up kit (MO BIO Laboratories, Inc., Carlsbad, CA). The purified PCR products were

169 quantified with a Qubit 2.0 and dsDNA BR Assay Kit (Invitrogen, Grand Island, NY) and an

170 equal mass (110 ng) of each was mixed into a single pool of all individuals and days. The no-

171 template control DNA was below detectable levels and thus the entire no-template reaction

172 mixture was added to the pool to be sequenced, to allow for detection of possible contaminants

173 (Salter et al., 2014). An ethanol precipitation was performed to concentrate the mixed pool of

174 index products. The DNA was sent to Selah Genomics (Greenville, SC) for Roche 454 next-

175 generation pyrosequencing. Sequence data from both axillae at day 3 are deposited in NCBI as

176 Bioproject PRJNA281417 (http://www.ncbi.nlm.nih.gov/bioproject/).

177 The resulting sequences were analyzed using the QIIME (version 1.7.0) microbial

178 community analysis software (Caporaso et al., 2010). This included initial filtering of DNA

179 sequences using default parameters (to insure their minimum and maximum lengths were $200 \mathrm{bp}$

180 and 1000 bp respectively, that quality score was at least 25, and that no ambiguous or

181 mismatched bases appeared in the primer sequence) and assignment of multiplexed reads to

182 samples based on their indexed barcode. Sequences from each sample were clustered into

183 Operational Taxonomic Units (OTUs) based on 97\% sequence similarity. Using a strategy of de

184 novo OTU picking in QIIME, a representative sequence was picked for each OTU and taxonomy

185 was assigned using the uclust consensus taxonomy classifier. Consistent with previous studies

186 (e.g., Hulcr et al., 2012) and with the default in QIIME, we assigned taxonomy to Level 6 (L6),

187 which assigns OTUs to genus level. Before performing any further analyses, we rarefied our data 
188 to 1000 reads per armpit sample. Four samples (only a single sample from each of four people

189 distributed across categories of product use, gender, and day) had fewer than 1000 reads and

190 were excluded from further analyses. In order to retain all participants in our study without

191 having unbalanced data, we randomly selected either left or right armpit for each person and

192 analyzed one armpit for day 3 and the same armpit for day 6. Random selection was done using

193 the RANDBETWEEN function in Microsoft Excel 2013, and selection of right versus left armpit

194 for each person is included in supplemental material (Supplemental Table 2). All subsequent

195 sequence based analyses were based on one armpit per person. Read counts of each OTU were

196 exported as a matrix for subsequent analyses after the removal of singletons and doubletons from

197 the dataset.

198 Sequence-based Analyses

199 For all participants we used day 3 and day 6 (for person T we had to use day 5 sample as

200 no day 6 sample existed) to assess richness (i.e., number of OTUs) and composition at the level

201 of each individual (number of OTUs per individual).

202 Richness: The program Primer-E v.6.1.15 (PRIMER-E, Plymouth, UK) was used to

203 calculate OTU richness (defined as number of OTUs present per person per day) based on the

204 OTU table. A mixed model ANOVA was performed using SPSS (version 21.0) to determine the

205 effect of day (early-day 3 vs. late-day 6, as described above), and product use (regular

206 antiperspirant users, deodorant users, or participants who did not regularly use underarm

207 products) on richness. The relationship between the abundance of culturable bacteria and

208 richness was computed across all groups using a Pearson rank correlation.

209 Composition: We visualized the composition of armpit bacteria using non-metric

210 multidimensional scaling ordination (NMDS) in Primer-E v.6.1.13 with PerMANOVA ext. 1.0.3 
211 (Clarke \& Gorley, 2006). To do this, we first constructed NMDS plots with 100 restarts and a

212 Type I Kruskal fit scheme based on a Dissimilarity matrix of Bray-Curtis distances. To assess the

213 relationship between product use and sampling period (early vs. late), we conducted a permuted

214 multivariate analysis of variance (PerMANOVA) test with treatment group (product use

215 categories described above) and sampling period and their interaction as factors, 9,999 iterations

216 and Type III sums of squares. We conducted SIMPER analyses for each significant factor to

217 determine the OTUs that contributed the most to pairwise between-group differences in

218 ordination space (Table 1). For a full comparison of all OTUs that differed in abundance between

219 the usage groups, we conducted a Metastats analysis (see

220 http://metastats.cbcb.umd.edu/detection.html and White et al., 2009) using pairwise comparisons

221 of average sequence reads for each product use group. Because Corynebacterium and

222 Staphylococcus have been previously shown to be important taxa in armpit communities (see

223 above), we conducted additional analyses of their abundances (i.e. number of reads) using a

224 mixed model ANOVA with the independent factors of product use, gender, day, and their

225 interaction. Finally, we determined the relationship between the abundance of Corynebacterium

226 and Staphylococcus across all groups using a Spearman rank correlation. We used SAS v. 9.3

227 Statistical Software (SAS Institute, Inc.) to conduct both of these analyses.

Results

230 Culture-based Results

231 If antiperspirants and deodorants negatively affect the abundance of bacteria in armpits,

232 we would expect that on the first day of no product use (Day 2 in our experiment) the residual

233 effects of products would lead to lower bacterial abundances in armpits of those individuals who 
234 use such products. As time progressed, we expect those abundances to increase among habitual

235 product users (as bacterial populations recover). Finally, once antiperspirant was applied to

236 (rebounded) armpit assemblages after five days of no product use, we expect abundances to

237 decline. In order to focus on one experimental treatment (product vs. no product use), we

238 instructed participants to alter only that aspect of their behavior.

239 Day 2-A significant effect of product use habit was observed on Day 2, the first day

240 none of the subjects applied product $\left(\mathrm{F}_{2,16}=3.9, \mathrm{p}<0.046\right.$; Table 2$)$. Subsequent tests performed

241 using Tukey HSD (Honestly Significant Difference) indicated that antiperspirant users who

242 ceased using product initially had significantly lower abundances of bacterial colonies on culture

243 plates than deodorant users who ceased using product $(\mathrm{p}<0.05)$. Significant pairwise differences

244 were not observed between antiperspirant users and subjects using no product, nor between

245 either product-use group and subjects who use no product.

246 Days 1-6 - With the disuse of antiperspirants and deodorants, bacterial abundance

247 significantly increased during the course of our experiment $\left(\mathrm{F}_{5,55}=4.92, \mathrm{p}=0.001\right)$ (Table 2).

248 Subsequent tests performed using Tukey HSD indicated that abundances on Day 5 were

249 significantly higher than on Day $1(p<0.05)$ and Day $2(p<0.05)$. Abundances on Day 4 were

250 significantly higher than on Day $1(\mathrm{p}<0.05)$. This increase over time was independent of product

251 use, gender, or any interactions among these variables.

252 Antiperspirant application (Day 6 versus 7-8)-Bacterial abundances on Day 7 (mean

$253 \mathrm{CFU} / \mathrm{cm}^{2} \pm$ standard deviation $\left.=3.1 \pm 8.4\right)$ and Day $8($ mean $=1.0 \pm 1.9)$, after antiperspirant

254 application, were an order of magnitude lower than on Day 6 (mean $=30.0 \pm 26.1$; overall $\mathrm{F}_{2,28}$

$255=19.0, \mathrm{p}<0.001)$.

256 Sequence-based Results 

more complete armpit bacterial communities (rather than just the abundance of culturable taxa), we analyzed the richness (i.e., OTU diversity) and composition of armpit microbes detected via sequencing of $16 \mathrm{~S}$ rDNA in participants sampled on two days: early (Day 3 of the experiment, 261 the second day of continued product disuse) and late (Day 6, the fifth day of continued product 262 disuse). On both of these days, the only differences we expected were those due to long-term 263 product use, which might occur due to differences in who chooses to use deodorant or antiperspirant (with individuals with more odorous microbial assemblages perhaps more likely to use product) or due to the product use itself. If the former, we would expect individuals with more product use to tend to be the same individuals with more slow-growing odor producing bacteria such as corynebacteria. If the latter, then we expected the reverse. passed the quality screens of the 454 platform and QIIME filtering. After rarefaction to 1000 reads per sample we observed a total of 106 OTUs of bacteria and Archaea in armpits of the 17 individuals in our study, with an average richness of 22 OTUs per person. Because Archaea were represented by just two OTUs found on the same person (Candidatus nitrososphaera and Halococcus), we hereafter focus on bacterial results. underarm product use, were more diverse (mean number of OTUs $\pm \mathrm{SD}=31.2 \pm 24.4$ ) than those of deodorant users (mean $\pm \mathrm{SD}=10.7 \pm 6.2$ ), or users of no product (mean $\pm \mathrm{SD}=20.5 \pm$ 13.4) (ANOVA, $F_{2,14}=3.91, p=0.045$, Figure 1, Supplemental Table 1, Supplemental Table 3 ). Subsequent Tukey HSD tests supported our finding that bacterial communities of antiperspirant users were significantly richer than those of deodorant users $(\mathrm{p}<0.05)$. No significant differences 
280 were observed between bacterial richness of either group of product-users and users of no

281 product. Neither a significant effect of day nor an interaction was observed. The number of

282 OTUs was not correlated with the abundance of culturable bacteria $(\mathrm{r}=-0.37, \mathrm{p}>0.05)$.

283 Composition: The composition of bacteria was strongly associated with underarm

284 product use (PerMANOVA, $P$ Treatment $=0.0001$, Figure 2), but not sampling period (where

285 compositional differences were measured as a function of the relative abundance of taxa based

286 on read number). The five bacterial OTUs that contributed most to differences between each pair

287 of product use groups based on a SIMPER analysis are shown in Table 1. These results were

288 largely consistent with those from a Metastats analysis (White et al. 2009), which indicated some

289 OTUs were significantly more abundant in specific product use groups (Supplemental Table 4).

290 Overall, the bacteria that contributed the most to differences between microbial assemblages

291 among product use groups were an OTU of Staphylococcaceae (although at the L6 level this

292 OTU was classified as "Staphylococcaceae_other", we expect it almost certainly is a

293 Staphylococcus) and an OTU of Corynebacterium. The common OTU of Staphylococcaceae was

294 reduced in participants who did not use underarm products compared to either deodorant users

295 (who had $>186 \%$ more of the Staphylococcaceae OTU; Table 1) or antiperspirant users (who had

$296>181 \%$ more of the Staphylococcaceae OTU; Table 1). Conversely, the common OTU of

297 Corynebacterium was most common in participants who did not use underarm products; they

298 had $>109 \%$ more Corynebacterium than participants who regularly used deodorant and $>335 \%$

299 more Corynebacterium than those who used antiperspirant (Table 1). We examined the evenness

300 of armpit microbes (See Supplemental Material), but there were no significant effects of

301 sampling time, our treatments, or their interaction on this metric of community structure.

302 To further examine patterns in the abundances of Staphylococcaceae and 
303 Corynebacterium, mixed model ANOVAs were performed to test for day and gender effects, and

304 interactive effects of these variables with product use on each of these two taxa, both of which

305 were important in our analyses, but are also known to be functionally important armpit taxa. An

306 effect of product was observed (Figure $3 \mathrm{a}, \mathrm{F}_{2,11}=8.36, \mathrm{p}=0.006$ ), such that the abundance of

307 the OTU of Staphylococcaceae was lower in participants who did not use underarm products

308 compared to users of antiperspirant (Tukey HSD, $p=0.005)$ or deodorant (Tukey HSD, $p=$

309 0.007), as expected based on results of the composition analysis (see mean abundance values in

310 Table 1). Conversely, users of no product had significantly higher abundances of

311 Corynebacterium than users of antiperspirant (Tukey HSD, $\mathrm{p}<0.001$ ) or deodorant (Tukey HSD,

$312 \mathrm{p}=0.006)$ (Figure 3b, $\mathrm{F}(2,11)=16.56, \mathrm{p}<0.001)$. Corynebacterium abundance tends to be

313 positively associated with the strength of body odors (Taylor et al., 2003). This pattern is the

314 opposite of what we would expect if the bacteria in the armpits of product users are different

315 from those of non-product users because product users are often individuals who have more

316 odorous biotas (Harker et al., 2014). No other significant main or interactive effects were

317 observed, including no effect of day on abundances of Staphylococcaceae or Corynebacterium.

318 Whereas Callewaert et al. (2013) found that females tended to be dominated by Staphylococcus

319 spp., and males by Corynebacterium species, we observed no effect of gender on abundances of

320 these two bacterial lineages.

321 As a result of the differential associations of these two bacterial taxa to product use, the

322 abundances of Staphylococcaceae and Corynebacterium were strongly negatively correlated to

323 each other across all individuals (Figure 4; Pearson Rank Correlation: $\mathrm{r}=-0.697, \mathrm{p}<0.0001$ ). 


\section{Discussion}

Overall, we found an initial negative effect of antiperspirant, but not deodorant, on

328

329

330

331

332

333

334

335

336

337

338

339

340

341

342

343

344

345

346

347

bacterial abundance using a traditional culture-based approach. After one day of ceasing product use, antiperspirant users had fewer colonies of culturable bacteria than deodorant users or users of no product. Colony abundance increased, particularly across Days 2-5, with continued product disuse. When all participants began to use antiperspirant, bacterial counts declined. Together these results demonstrate, as expected, that antiperspirants are capable of strongly reducing the biomass of the armpit microbial community, largely independent of the historic product use of individuals. In short, antiperspirant appears to have a clear negative effect on bacterial abundance, one that can be detected in individuals using antiperspirant and that can be produced experimentally. The effect of deodorant on bacterial abundance is more modest, if present at all. These results were not unexpected, in light of two key differences between deodorants and antiperspirants: 1) many deodorants are ethanol-based and likely more water soluble and easier to wash away than antiperspirants; and 2) antiperspirants contain aluminum-based salts that reduce sweat by forming precipitates that physically block sweat glands (Benohanian, 2001) and thus may reduce resources necessary for the growth of microbial communities.

A key question though, given the relative difficulty of culturing many axillary bacteria, particularly those that are slow-growing, is the extent to which changes in the abundance of easily cultured bacteria in response to a short-term experiment match the differences in the entire assemblage, when evaluated using more comprehensive sequencing approaches, particularly differences seen in association with long-term use. Here, several practical challenges exist. Long term experiments on deodorant and antiperspirant, experiments conducted over years, require very committed participants. They are also, however, ethically questionable if prolonged use of 
349 deodorants and antiperspirants can have persistent effects on microbial assemblages that in turn, 350 affect health and well-being. In addition, it has proven very challenging to isolate DNA from

351 participants actively using underarm products. Our approach to dealing with this challenge was

352 to use sequence-based approaches to consider the assemblages of microbes in armpits

353 immediately after product use was ceased (as a measure of long-term differences among

354 individuals differing in product use, with only a short period for any shift post disuse). In

355 addition, we considered samples from several days later, once some shift might have been able to

356 occur. These sequence-based data are largely correlational rather than experimental and yet

357 allow strong inference when coupled with experimental data on easily culturable microbes.

358 Based on sequencing of $16 \mathrm{~S}$ rDNA, long-term antiperspirant users tended to have more

359 bacterial OTUs in their armpits (multiple days after ceasing product use) than did long-term

360 deodorant users (after disuse of product; Figure 1). We expected that application of underarm

361 products would negatively affect dominant species, thereby creating more opportunities for rare

362 species to become established. However, we did not observe this effect in deodorant users, who

363 actually had fewer species of bacteria in their armpits compared to armpits of participants who

364 use no product (Figure 1). Our findings are consistent with those of Callewaert et al. (2014). In

365 line with Callewaert et al.'s (2014) comparison of individuals, we observed a larger change (i.e.

366 increased community richness) in the armpit microbial community when regular product users

367 stopped wearing and then resumed use of antiperspirants, compared to those asked to stop then

368 resume use of deodorants. As such, we expect that our results represent a general effect of

369 antiperspirant use. We can only speculate as to why effects of stopping long-term deodorant and

370 antiperspirant use might have such disparate effects, though note that the particular antiperspirant

371 products (i.e., brands) our participants reported to us all contain aluminum salts. These 
372 compounds may alter the underarm habitat (in a manner that deodorants do not), and provide a

373 selective advantage to bacteria not historically common in the human armpit habitat. Based on

374 our study, this underarm habitat alteration lasts multiple days after stopping product use (see

375 Figure 1, which is based on data from days 2 and 5). The highly abundant microbes identified

376 here compare reasonably well to other studies analyzing moist areas of human skin showing high

377 abundance of Staphylococcaceae and Corynebacteriaceae, among others (see Grice and Segre,

3782011 for a review).

379 The composition of armpit bacterial communities of both antiperspirant and deodorant

380 users was associated with differences in abundances of the two most abundant bacterial taxa, an

381 OTU of Staphylococcaceae and an OTU of Corynebacterium, relative to users of no product.

382 The Staphylococcaceae OTU was the most dominant bacterial group in participants ceasing

383 antiperspirant and deodorant use, followed by the Corynebacterium OTU, whereas this

384 dominance order was reversed among users of no product. In our sequence-based study, we

385 cannot preclude the possibility that individuals who use deodorant and antiperspirant tend to

386 have non-random assemblages of armpit bacteria. But we would expect that, if anything, such

387 individuals would tend to have more odoriferous assemblages of microbes. Armpit odor is

388 largely associated with Corynebacterium, such that we would then expect more

389 Corynebacterium in product users. We found the opposite.

390 Unlike many taxa on the body, these two taxa have been relatively well characterized

391 with regard to their biology. Species of Corynebacterium are associated with the dominant odors

392 of the armpits and individuals with more Corynebacterium are likely to have stronger body odor

393 (Taylor et al., 2003). Ceasing the use of deodorant and antiperspirant was associated with lower

394 levels of Corynebacterium, in line with expectations, given that companies that sell underarm 
395 products aim to reduce body odor through reduction in overall bacterial counts.

396 We recognize that two additional considerations may have affected our results and those

397 of other studies of armpits. First, because as much as $90 \%$ of the bacterial OTUs identified

398 through DNA-sequence based surveys such as ours are bacterial taxa that typically cannot be

399 cultured under standard laboratory conditions, we chose to culture bacteria to inform when to

400 conduct our sequence based analyses (i.e., to determine if residual product impaired colony

401 growth), and to make general comparisons across product use groups. As such, we used standard

402 LB plates grown under aerobic conditions, which like all media, only allow the culturing of a

403 subset of lineages. Although this did not affect the overall conclusions of our sequence-based

404 results, this may have affected the abundances, in that perhaps those bacteria that were abundant

405 in the armpits of non-product users (e.g., Corynebacterium) were not those easily grown on LB

406 agar. However, this does not account for the increased abundance of culturable bacteria in non-

407 product users from days 1 to 4 , which is either due to chance and small sample size, or some

408 systemic change that applied to all of our participants.

409 Second, in comparing the armpit communities of product-users versus non-product users,

410 we expect that non-product users began our study with more stable armpit communities than

411 those of product-users who recently ceased using product, a standard feature of press

412 experiments, which are common in ecology. Press experiments are designed to understand

413 whether the application of some treatment and then its removal have similar effects (powerful

414 evidence for the influence of the treatment). However, all press experiments provide direct

415 evidence about the experimental effect for the time interval of the study. Our focus was on eight

416 days, sufficient time for many generations of bacteria. It is very possible that were our

417 experiment shorter or longer that our results might have been different. The armpit is a dynamic 
418 system and future studies might usefully follow-up with longer term experiments, though only

419 after careful consideration of the ethics of such experiments given that the bacteria whose

420 composition is altered by deodorant and antiperspirant are of direct health consequences

421 (Christensen \& Brüggemann, 2014).

422 A larger sample size would allow us to test hygiene effects of washing frequency and

423 soap type (as these may potentially disturb armpit communities, even of non-product users) as

424 well as additional demographic factors, such as age and gender. This latter point will be

425 informative as gender differences between Staphylococcus and Corynebacterium have been

426 noted in other studies (Callewaert et. al. 2013) and will be informative to tease apart gender

427 biases from our product use categories.

428

429

430

431

432

433

434

435

436

437

438

439

440

\section{Conclusions}

Although it has long been recognized that skin bacterial composition varies strongly among individuals, accounting for such variation has been a challenge, one that has led some authors to suggest that the composition of the skin biome might simply be stochastic, a function of chance colonizations and unpredictable dynamics. Here, we find that the composition of the armpit microbiome is highly predictable, being dominated by Staphylococcaceae and Corynebacterium, and strongly influenced by product use. Species of the Staphylococcaceae include beneficial symbionts (Rosenthal et al., 2011; Christensen \& Brüggemann, 2014) but also dangerous pathogens (Otto, 2009; Ryu et al., 2014). It is noteworthy in this regard that the armpit is a common site for pathogenic MRSA infections in athletes (Cohen, 2008). However, we cannot discern which of these taxa are being favored with product use based on our data. The broader health consequences of antiperspirant and deodorant use are not well studied. 
441 Although it has been suggested that deodorant and/or antiperspirant use is associated with

442 incidence or age of breast cancer diagnosis (McGrath, 2003), support for this association is 443 equivocal at best (Hardefeldt, Edirimanne \& Eslick, 2013). Whether antiperspirant or deodorant

444 tends to favor less beneficial or even pathogenic bacterial species does not seem to have ever

445 been considered. Recent work indicates that the microbial community structure of the skin,

446 including its commensal/symbiotic residents, exerts significant influence on human health and

447 disease, particularly in the emergence of pathogenic strains of Staphylococcus aureus, $S$.

448 epidermidis, and Propionibacterium acnes (Otto, 2009; Rosenthal et al., 2011; Christensen \&

449 Brüggemann, 2014). Rosenthal et al. (2011) hypothesized that the skin microbiome may be "an

450 antibiotic resistance reservoir," as has been shown to be the case with the human gut microbiome

451 (Sommer, Dantas \& Church, 2009). Our work clearly demonstrates that antiperspirant use

452 strikingly alters armpit bacterial communities, making them more species rich. Because

453 antiperspirants only came into use within the last century, we presume that the species of bacteria

454 they favor are not those historically common in the human armpit. Whether these species may

455 interfere with the function of beneficial skin symbionts, contribute antibiotic resistance genes,

456 prove benign, or perhaps even confer beneficial effects to human health remains an intriguing

457 avenue for further study. 


\section{Acknowledgements}

460 We thank Greg Pahel for assistance with laboratory work. We thank all of the dedicated citizen 461 scientist volunteers who participated in our study. We also thank the reviewers of our manuscript 462 for their time and insight.

463

464 
465

466

467

468

469

470

471

472

473

474

475

476

477

478

479

480

481

482

483

484

485

486

487

488

\section{References}

Benohanian A. 2001. Antiperspirants and deodorants. Clinics in Dermatology 19:398-405.

Callewaert C, Hutapea P, Van de Wiele T, Boon N. 2014. Deodorants and antiperspirants affect the axillary bacterial community. Archives of Dermatological Research 306:701-710.

Callewaert C, Kerckhof FM, Granitsiotis MS, Gele MV. 2013. Characterization of Staphylococcus and Corynebacterium clusters in the human axillary region. PLOS ONE 8(8):e70538.

Caporaso JG, Kuczynski J, Stombaugh J, Bittinger K, Bushman FD, Costello EK, Fierer N, Peña AG, Goodrich JK, Gordon JI, Huttley GA, Kelley ST, Knights D, Koenig JE, Ley RE, Lozupone CA, McDonald D, Muegge BD, Pirrung M, Reeder J, Sevinsky JR, Turnbaugh PJ, Walters WA, Widmann J, Yatsunenko T, Zaneveld J, Knight R. 2010. QIIME allows analysis of high-throughput community sequencing data. Nature Methods doi: 10.1038/nmeth.f.303.

Caporaso JG, Lauber CL, Costello EK, Berg-Lyons D, Gonzalez A, Stombaugh J, Knights D, Gajer P, Ravel J, Fierer N, Gordon, JI, Knight, N. 2011. Moving pictures of the human microbiome. Genome Biology 12:R50.

Christensen GJM, Brüggemann H. 2014. Bacterial skin commensals and their role as host guardians. Beneficial Microbes 5(2):201-215.

Clarke KR, Gorley RN. 2006. PRIMER v6: User Manual/Tutorial. Plymouth: PRIMER-E.

Cohen PR. 2008. The skin in the gym: a comprehensive review of the cutaneous manifestations of community-acquired methicillin resistant Staphylococcus aureus infection in athletes. Clinics in Dermatology 26:16-26.

Costello EK, Lauber CL, Hamady M, Fierer N, Gordon FI, Knight R. 2009. Bacterial community 
variation in human body habitats across space and time. Science 326:1694-1697.

490 Egert M, Schmidt I, Höhne HM, Lachnit T, Schmitz RA, Breves R. 2011. rRNA-based profiling

491

492

493

494

495

496

497

498

499

500

501

502

503

504

505

506

507

508

509

510

511 of bacteria in the axilla of healthy males suggests right-left asymmetry in bacterial activity. FEMS Microbial Ecology 77:146-153.

Eichenwald HF, Shinefield HR, Boris M, Ribble JC. 1965. "Bacterial interference" and staphylococcic colonization in infants and adults. Annals of the New York Academy of Sciences 128(1):365-80.

Flores, G. E., Henley, J. B., Fierer, N. 2012. A direct PCR approach to accelerate analyses of human-associated microbial communities. PlosOne 7(9):e44563.

Fredrich E, Barzantny H, Brune I, Tauch A. 2013. Daily battle against body odor: towards the activity of the axillary microbiota. Trends in Microbiology 21(6):305-312.

Gao Z, Perez-Perez GI, Chen Y, Blaser MJ. 2010. Quantitation of major human cutaneous bacterial and fungal populations. Journal of Clinical Microbiology 48(10):3575-3581.

Grice EA, Kong HH, Conlan S, Deming CB, Davis J, Young AC, NISC Comparative Sequencing Program, Bouffard GG, Blakesley RW, Murray PR, Green ED, Turner ML, Segre JA. 2009. Topographical and temporal diversity on the human skin microbiome. Science 324:1190-1192.

Grice EA, Segre JA. 2011. The skin microbiome. Nature Reviews Microbiology 9:244-253.

Hardefeldt PJ, Edirimanne S, Eslick GD. 2013. Deodorant use and breast cancer risk. Epidemiology 24(1):172.

Harker, M., Carvell AM, Marti VP, Riazanskaia S, Kelso H, Taylor D, Grimshaw S, Arnold DS, Zillmer R, Shaw J, Kirk JM, Alcasid ZM, Gonzales-Tanon S, Chan GP, Rosing EA, Smith, AM. 2014. Functional characterisation of a SNP in the $A B C C 11$ allele - effects on 
axillary skin metabolism, odour generation and associated behaviours. J Dermatol Sci 73:

513 23-30.

514 Hulcr J, Latimer AM, Henley JB, Rountree NR, Fierer N, Lucky A, Lowman MD, Dunn RR. 2012. A jungle in there: bacteria in belly buttons are highly diverse, but predictable. PLOS ONE 7(11):e47712.

517 Kong HH, Segre JA. 2012. Skin microbiome: Looking back to move forward. Journal of Investigative Dermatology 132:933-939.

Leyden JJ, McGinley KJ, Hölzle E, Labows JN, Kligman AM. 1981. The microbiology of the human axilla and its relationship to axillary odor. Journal of Investigative Dermatology 77:413-416.

Marples MJ. 1965. The Ecology of the Human Skin. Springfield, IL: Charles C. Thomas, Bannerstone House.

McGrath KG. 2003 An earlier age of breast cancer diagnosis related to more frequent use of antiperspirants/deodorants and underarm shaving. European Journal of Cancer Prevention 12(6):479-485.

Otto M. 2009. Staphylococcus epidermidis -- the 'accidental' pathogen. Nature Reviews Microbiology 7:555-567.

Rasband WS. 1997-2014. ImageJ. US National Institutes of Health, Bethesda, Maryland, USA, Available at http://imagej.nih.gov/ij/.

Rosenthal M, Goldberg D, Aiello A, Larson E, Foxman B. 2011. Skin microbiota: Microbial community structure and its potential association with health and disease. Infection, Genetics, and Evolution 11:839-848.

534 Ryu S, Song PI, Seo CH, Cheong H, Park Y. 2014. Colonization and infection of the skin by $S$. 
aureus: Immune system evasion and the response to cationic antimicrobial peptides. International Journal of Molecular Sciences 15:8753-8772.

537 Salter S, Cox MJ, Turek EM, Calus ST, Cookson WO, Moffatt MF, Turner P, Parkhill J, Loman

538 N, Walker AW. 2014. Reagent contamination can critically impact sequence-based microbe analyses. bioRxiv doi: 10.1101/007187.

Shelley WB, Hurley HJ, Nicholas AC. 1953. Axillary odor: Experimental study of the role of bacteria, apocrine sweat and deodorants. Archive für Dermatologie und Syphilis-G $68: 430$.

543

544

545

546

547

548

549

550

551

552

553

554

Sommer MOA, Dantas G, Church GM. 2009. Functional characterization of the antibiotic resistance reservoir in the human microflora. Science 325:1128-1131.

Taylor D, Daulby A, Grimshaw S, James G, Mercer J, Vaziri S. 2003. Characterization of the microflora of the human axilla. International Journal of Cosmetic Science 25:137-145.

White, J.R., Nagarajan, N., Pop, M. 2009. Statistical methods for detecting differentially abundant features in clinical metagenomic samples. PLoS Comput. Biol. 5, e1000352.

Wilke K, Martin A, Terstegen L, Biel SS. 2007. A short history of sweat gland biology. International Journal of Cosmetic Science 29:169-179. 


\section{Figure 1 (on next page)}

Mean composition and richness of bacterial OTUs

Mean composition and richness of bacterial OTUs for all three product user types, combined OTU data from two and five days after stopping product use. Bacteria with greater than 10 sequence reads across all users in each category are shown. The top three bacterial OTUs are shown; a full list is available in Supplemental Table 1. Antiperspirant users have much richer armpits (22\% other bacteria versus $5 \%$ for deodorant users and $9 \%$ for no product users). At the L6 level of OTU assignment, the OTU for the highly abundant Staphylococcaceae was "Staphylococcaceae_other" indicating that the genus was unassigned. We refer to this OTU for simplicity throughout the remainder of the figures and text as Staphylococcaceae, but that this does represent one group within Staphylococcaceae and does not denote all identified OTUs in this family. 


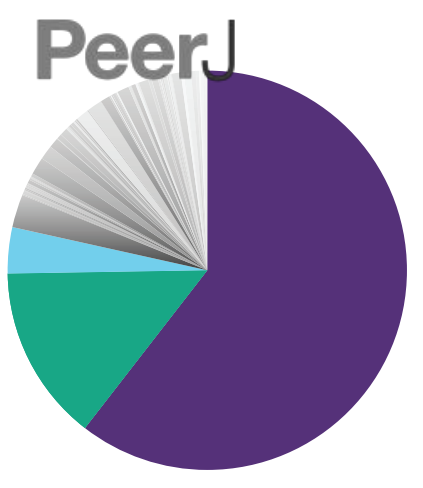

- Staphylococcaceae 60\%

- Corynebacterium 14\%

- Anaerococcus 4\%

10 Other $22 \%$

Ceased

Antiperspirant use

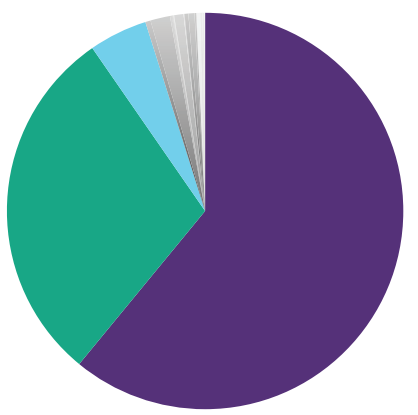

- Staphylococcaceae 61\%

- Corynebacterium 29\%

- Anaerococcus 5\%

1] Other 5\%

Ceased

Deodorant use

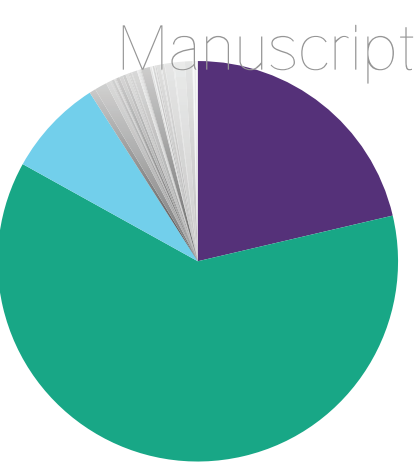

- Staphylococcaceae 21\%

- Corynebacterium 62\%

- Anaerococcus 8\%

10 Other $9 \%$

No

Product use 


\section{Figure 2 (on next page)}

Non-metric multidimensional scaling plot of armpit microbes

Non-metric multidimensional scaling plot of armpit microbes based upon rarefaction using 1000 sequence reads. Small symbols represent individuals from each treatment group and large symbols represent group centroids $\pm 1 \mathrm{SE}$. 


\section{Rarefied Abundance NMDSeerJ}

2D Stress $=0.07$

PerMANOVA: Previous Product Use $P=0.0001$

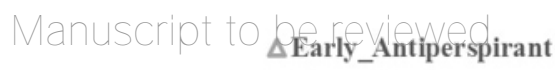

$\square$ Early_Deodorant

OEarly_None

$\Delta$ Late_Antiperspirant

口Late_Deodorant

OLate_None

$\Delta$

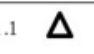

$\Delta$

(1)

$\Delta$

$\Delta \triangle \square$

口

○

Axis 1

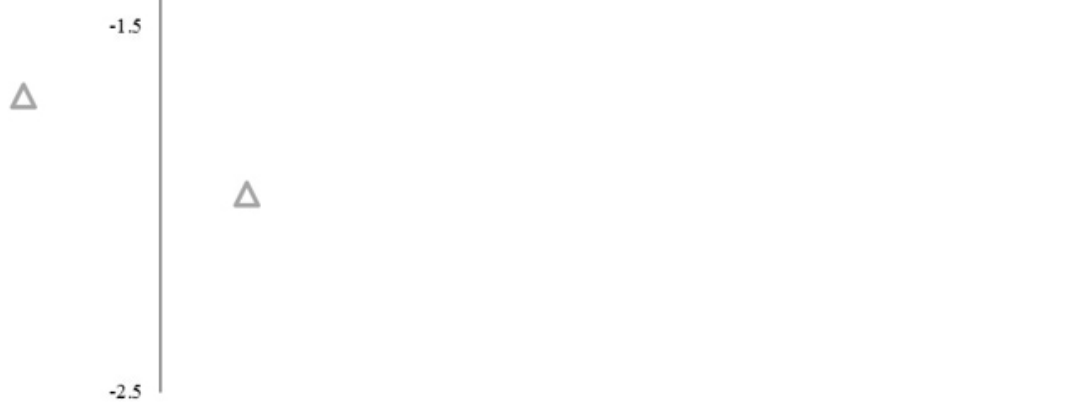


Figure 3 (on next page)

Mean abundances of Staphylococcaceae and Corynebacterium across product use groups

Mean abundances of (a) Staphylococcaceae and (b) Corynebacterium of participants who regularly used antiperspirant, deodorant or no underarm products based upon sequence data. Underarm product use significantly affected the abundance of both Staphylococcaceae and Corynebacterium (2-way ANOVA: $p<0.0001$ for both microbes). However, neither sampling period nor its interaction with product use significantly affected either microbe (2way ANOVA: $p>0.05$ ). 

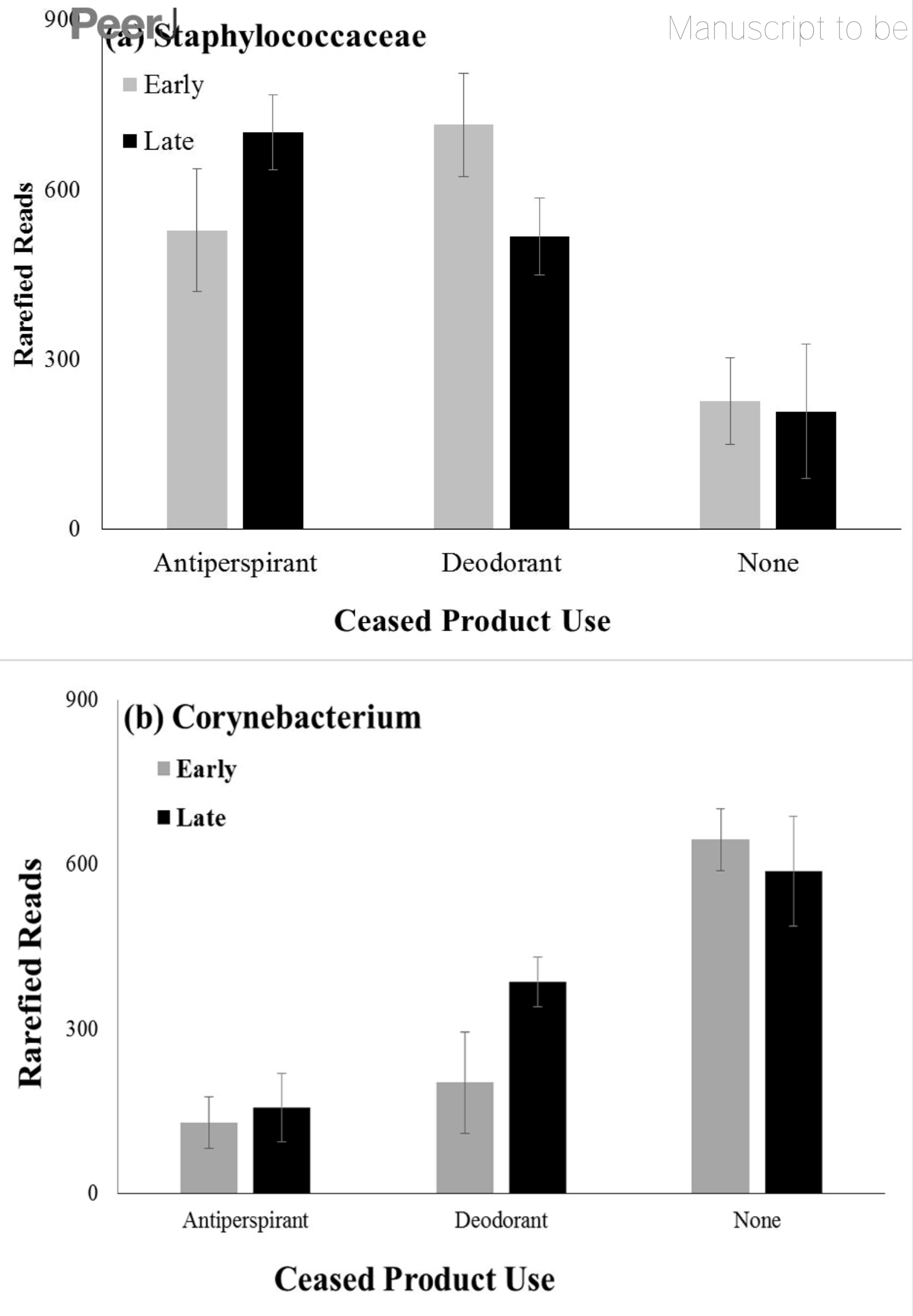
Figure 4 (on next page)

Relationship between the abundances of Staphylococceae and Corynebacterium across all individuals.

Spearman Rank Correlation: $r=-0.697, p<0.0001$. 


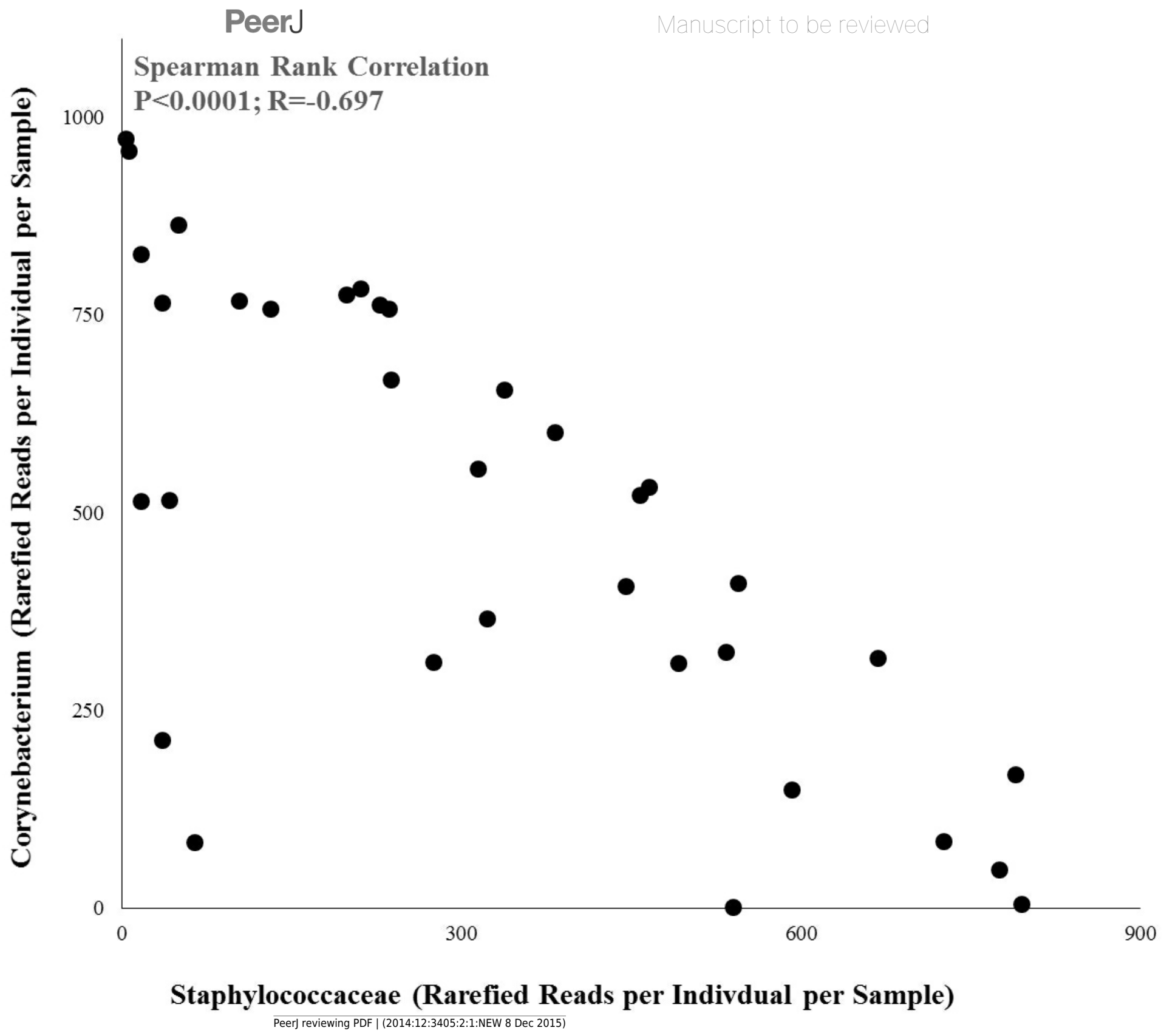




\section{Table $\mathbf{1}$ (on next page)}

Comparisons of the average abundances and \% contributions for the top 5 microbes that contributed the most to differences between each pair of treatment groups.

*In a post-hoc test, participants ceasing antiperspirant use and participants ceasing deodorant use were not significantly different from each other $(P=0.095)$. 
1 Table 1: Comparisons of the average abundances and \% contributions for the top 5 microbes that contributed the most to differences between each 2 pair of treatment groups.

\begin{tabular}{|c|c|c|c|c|c|}
\hline \multirow{2}{*}{ Comparison } & \multirow{2}{*}{ Family } & \multirow{2}{*}{ Genus } & \multicolumn{2}{|c|}{ Average Abundance } & \multirow{2}{*}{$\begin{array}{c}\% \text { Contribution } \\
\text { to Differences }\end{array}$} \\
\hline & & & Antiperspirant & Deodorant & \\
\hline \multirow{6}{*}{ Antiperspirant vs. Deodorant* } & Staphylococcaceae & Other & 600.36 & 609.00 & 32.91 \\
\hline & Corynebacteriaceae & Corynebacterium & 141.29 & 293.80 & 27.69 \\
\hline & Clostrididaceae & Anaerococcus & 37.07 & 47.60 & 8.41 \\
\hline & Alicyclobacillaceae & Alicyclobacillus & 26.57 & 4.30 & 3.41 \\
\hline & Clostrididaceae & Finegoldia & 0.86 & 16.00 & 2.05 \\
\hline & & & None & Deodorant & \\
\hline \multirow{6}{*}{ None vs. Deodorant } & Staphylococcaceae & Other & 212.90 & 609.00 & 43.63 \\
\hline & Corynebacteriaceae & Corynebacterium & 615.90 & 293.80 & 35.36 \\
\hline & Clostrididaceae & Anaerococcus & 78.50 & 47.60 & 8.74 \\
\hline & Clostrididaceae & Finegoldia & 9.90 & 16.00 & 2.16 \\
\hline & Camplobacteraceae & Camplobacter & 8.60 & 0.90 & 0.94 \\
\hline & & & None & Antiperspirant & \\
\hline \multirow{5}{*}{ None vs. Antiperspirant } & Corynebacteriaceae & Corynebacterium & 615.90 & 141.29 & 37.82 \\
\hline & Staphylococcaceae & Other & 212.90 & 600.36 & 34.21 \\
\hline & Clostrididaceae & Anaerococcus & 78.50 & 37.07 & 6.98 \\
\hline & Alicyclobacillaceae & Alicyclobacillus & 5.10 & 26.57 & 2.02 \\
\hline & Porphyromonadaceae & Porphyromonas & 2.70 & 14.07 & 1.22 \\
\hline
\end{tabular}


3 *In a post-hoc test, participants ceasing antiperspirant use and participants ceasing deodorant use were not significantly different from 4 each other $(\mathrm{P}=0.095)$. 


\section{Table 2 (on next page)}

Mean (standard deviation) of abundance of culturable bacteria (CFU/ $\left.\mathrm{cm}^{2}\right)$ on culture plates at baseline (Day 1, when all subjects followed regular habit of product use application) and subsequent days (Days 2-6) when all subjects ceased usage of $u$

Abundances on Day 5 were significantly higher than on Days 1 and 2. Abundances on Day 4 were significantly higher than on Day 1 . Abundances were measured by resuspending skin microbes in PBS solution and then plating 1/25th of the volume on culture plates. 
Table 2: Mean (standard deviation) of abundance of culturable bacteria (CFU/ $\mathrm{cm}^{2}$ ) on culture plates at baseline (Day 1, when all subjects followed regular habit of product use application) and subsequent days (Days 2-6) when all subjects ceased usage of underarm products and (Days 7 and 8 ) when all subjects used underarm product provided by us. A significant effect of product use was shown on Day $2\left(\mathrm{~F}_{2,16}=3.9, \mathrm{p}=0.046\right)$.

\begin{tabular}{|c|c|c|c|c|c|c|c|c|}
\hline & Day 1 & Day 2 & Day 3 & Day 4 & Day 5 & Day 6 & Day 7 & Day 8 \\
\hline $\begin{array}{l}\text { Antiperspirant } \\
\text { users }(4 \mathrm{~F}, 3 \mathrm{M})\end{array}$ & $\begin{array}{c}4.1 \\
(7.2) \\
\end{array}$ & $\begin{array}{c}6.4 \\
(3.8) \\
\end{array}$ & $\begin{array}{c}8.0 \\
(12.1) \\
\end{array}$ & $\begin{array}{c}18.4 \\
(30.3) \\
\end{array}$ & $\begin{array}{c}36.1 \\
(28.1) \\
\end{array}$ & $\begin{array}{c}22.2 \\
(22.2) \\
\end{array}$ & $\begin{array}{c}5.1 \\
(13.1) \\
\end{array}$ & $\begin{array}{c}1.6 \\
(2.4) \\
\end{array}$ \\
\hline $\begin{array}{c}\text { Deodorant } \\
\text { users }(2 \mathrm{~F}, 3 \mathrm{M})\end{array}$ & $\begin{array}{c}17.2 \\
(31.7) \\
\end{array}$ & $\begin{array}{c}25.8 \\
(18.8) \\
\end{array}$ & $\begin{array}{c}42.1 \\
(43.5)\end{array}$ & $\begin{array}{c}46.5 \\
(31.9) \\
\end{array}$ & $\begin{array}{c}41.3 \\
(32.4)\end{array}$ & $\begin{array}{c}46.0 \\
(36.9) \\
\end{array}$ & $\begin{array}{c}3.3 \\
(3.1) \\
\end{array}$ & $\begin{array}{c}0.9 \\
(1.9)\end{array}$ \\
\hline $\begin{array}{c}\text { No product } \\
\text { users }(2 \mathrm{~F}, 3 \mathrm{M})\end{array}$ & $\begin{array}{r}7.2 \\
(8.0) \\
\end{array}$ & $\begin{array}{c}17.5 \\
(11.9) \\
\end{array}$ & $\begin{array}{c}23.9 \\
(15.3) \\
\end{array}$ & $\begin{array}{c}28.2 \\
(24.8)\end{array}$ & $\begin{array}{c}24.0 \\
(19.1)\end{array}$ & $\begin{array}{c}25.1 \\
(12.7)\end{array}$ & $\begin{array}{c}0.0 \\
(0.0)\end{array}$ & $\begin{array}{c}0.1 \\
(0.2)\end{array}$ \\
\hline $\begin{array}{l}\text { All groups } \\
\text { combined }\end{array}$ & $\begin{array}{c}8.9 \\
(17.9)\end{array}$ & $\begin{array}{c}15.4 \\
(14.1)\end{array}$ & $\begin{array}{c}22.7 \\
(28.3)\end{array}$ & $\begin{array}{c}29.5 \\
(29.9)\end{array}$ & $\begin{array}{c}34.1 \\
(26.5)\end{array}$ & $\begin{array}{c}30.0 \\
(26.1)\end{array}$ & $\begin{array}{c}3.1 \\
(8.4)\end{array}$ & $\begin{array}{c}1.0 \\
(1.9)\end{array}$ \\
\hline
\end{tabular}

4 Abundances on Day 5 were significantly higher than on Days 1 and 2. Abundances on Day 4 were significantly higher than on Day 1.

5 Abundances were measured by resuspending skin microbes in PBS solution and then plating $1 / 25$ th of the volume on culture plates. 